\title{
Effects of Wandering on the Coalescence of Black Hole Binaries in Galactic Centers
}

\section{Citation}

Chatterjee, Pinaki, Lars Hernquist, and Abraham Loeb. 2003. "Effects of Wandering on the Coalescence of Black Hole Binaries in Galactic Centers." The Astrophysical Journal 592 (1): 3241. https://doi.org/10.1086/375552.

\section{Permanent link}

http://nrs.harvard.edu/urn-3:HUL.InstRepos:41381793

\section{Terms of Use}

This article was downloaded from Harvard University's DASH repository, and is made available under the terms and conditions applicable to Other Posted Material, as set forth at http:// nrs.harvard.edu/urn-3:HUL.InstRepos:dash.current.terms-of-use\#LAA

\section{Share Your Story}

The Harvard community has made this article openly available.

Please share how this access benefits you. Submit a story.

Accessibility 


\title{
Effects of Wandering on the Coalescence of Black Hole Binaries in Galactic Centers
}

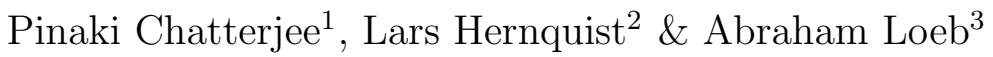 \\ Harvard-Smithsonian Center for Astrophysics, 60 Garden Street, Cambridge, MA 02138
}

\begin{abstract}
We examine whether massive binary black holes in spherically symmetric bulges of galaxies can achieve coalescence through the emission of gravitational radiation under the action of stellar dynamical processes alone. In particular, we address the importance of the Brownian motion of a binary's center-of-mass to its continued interaction with stellar orbits that allows it to keep hardening: the restoring force holding the binary at the center decreases as it depletes the central stellar density, causing the amplitude of wandering to increase. We use an analytical model and N-body simulations to calculate the time required to reach the gravitational-radiation dominated stage. We find that a substantial fraction of all massive binaries in galaxies can coalesce within a Hubble time.
\end{abstract}

Subject headings: black hole physics — galaxies: kinematics and dynamics celestial mechanics — galaxies: nuclei — methods: N-body simulations

\section{Introduction}

Observations indicate that most galaxies harbor supermassive black holes (BHs) at their centers (e.g., Magorrian et al. 1998). Since galaxy mergers are inevitable, there arises the question of the dynamical fate of the binary black holes (BBHs) that result from these mergers. This problem was first addressed by Begelman, Blandford \& Rees (1980) (for recent work, see, e.g., Milosavljević \& Merritt 2001, 2002, Yu 2002). Dynamical friction

\footnotetext{
${ }^{1}$ pchatterjee@cfa.harvard.edu

2lars@cfa.harvard.edu

${ }^{3}$ Guggenheim fellow. On sabbatical leave at the Institute for Advanced Study, Princeton, NJ 08540; loeb@ias.edu.
} 
causes the black holes in the original galaxies to sink to the center of the merged galaxy, and then become bound in a binary system. The semi-major axis of the binary, $a$, continues to shrink owing to dynamical friction until it reaches the point at which the binary becomes hard: $a_{h} \simeq G m_{2} / 4 \sigma^{2}$, where $\sigma$ is the one-dimensional stellar velocity dispersion, $G$ is the gravitational constant, and $m_{2}\left(<m_{1}\right)$ is the mass of the lighter of the two black holes (following Quinlan 1996). After this stage, the dominant mechanism by which the binary shrinks and loses energy is by three-body interactions with stars that pass within a distance $\sim a$ of the center of mass of the binary. The binary transfers energy to the stars by ejecting them at a much higher velocity. Once it shrinks to the point at which energy loss to gravitational waves becomes dominant, the binary can quickly coalesce.

Begelman, Blandford \& Rees (1980) conjectured that hardening of a tight binary by three-body interactions would continue only until it had interacted with all the stars contained within its loss cone (Frank \& Rees 1976); if this was not sufficient to drive it to the gravitational wave emission stage, the binary would stall at a certain separation, and harden very slowly at the two-body relaxation timescale (the timescale at which the loss cone can be repopulated), which turns out for many actual galaxies to be longer than the Hubble time. The lack of observational evidence for the existence of massive BBHs has led to suggestions that BBH merging may be facilitated by other processes, such as the loss of angular momentum to gas (e.g., Begelman, Blandford \& Rees 1980, Gould \& Rix 2000, Armitage \& Natarajan 2002), or triaxiality of the host galaxy (Yu 2002).

It is therefore of interest to inquire whether stellar dynamical processes alone would be sufficient to drive the BBH to the gravitational wave emission stage. Quinlan \& Hernquist (1997) pointed out the importance of the wandering of the binary to its hardening. In this paper, we investigate the shrinking of a hard black hole binary in a spherically symmetric galaxy, and ask particularly how Brownian motion of its center of mass can contribute to its continued hardening beyond the stage of loss cone evacuation. In $\S 2$, we set up an analytic model that appears to account for the results of N-body simulations described in $\S 3$. We comment on the impact of $\mathrm{BBH}$ wandering in $\S 4$, and derive characteristics of the merger in $\S 5$, on the assumption that hardening proceeds at the rate deduced in $\S 2$ and $\S 3$. We summarize our findings in $\S 6$.

\section{The Model}

Consider two black holes of masses $m_{1}$ and $m_{2}$. Let $\mu_{12}=m_{1} m_{2} /\left(m_{1}+m_{2}\right)$ be the reduced mass of this $\mathrm{BBH}$ system. Also, let $m_{2}$ be the lighter of the two BHs, with the mass ratio being $q=m_{2} / m_{1} \leq 1$. 
For illustrative purposes, we take the host cluster of stars to be described by a Plummer model of total mass $M$ and length parameter $r_{0}$. Thus, the density and potential profiles are given, respectively, by

$$
\begin{gathered}
\rho(r)=\frac{3 M r_{0}^{2}}{4 \pi} \frac{1}{\left(r^{2}+r_{0}^{2}\right)^{5 / 2}}, \\
\Phi(r)=-\frac{G M}{\left(r^{2}+r_{0}^{2}\right)^{1 / 2}}
\end{gathered}
$$

where $G$ is the gravitational constant and $r$ is the radial distance from the center of the stellar system, which is taken as the origin. In this case, the one-dimensional stellar velocity distribution in the core is given by $\sigma^{2}=G M / 6 r_{0}$.

The phase space distribution function of the stellar system, $f$, depends in general both on position $\vec{r}$, and stellar velocity $\overrightarrow{v_{a}}$, and is defined such that $f\left(\vec{r}, \overrightarrow{v_{a}}\right) d^{3} \vec{r} d^{3} \overrightarrow{v_{a}}$ is the mass in stars in the phase space volume $d^{3} \vec{r} d^{3} \overrightarrow{v_{a}}$. For the spherically symmetric Plummer model, we assume $f$ to be a function of the relative energy per unit mass $\mathcal{E}$ only (and independent of specific angular momentum), where $\mathcal{E}=-\frac{1}{2} v_{a}^{2}-\Phi(r)=\Psi(r)-\frac{1}{2} v_{a}^{2}, \Psi(r)=-\Phi(r)$ being the relative potential. The distribution function is then (e.g., Binney \& Tremaine 1987)

$$
f(\mathcal{E})=\frac{96}{7 \sqrt{8} \pi^{3}} \frac{M r_{0}^{2}}{(G M)^{5}} \mathcal{E}^{7 / 2} .
$$

Consider an encounter between the $\mathrm{BBH}$ and a star of mass $m_{*}$. Let the impact parameter be $p$ and the initial relative velocity (when the star is at infinity) be $V_{0}$. If $r_{\text {min }}$ is the minimum distance reached by the star to the $\mathrm{BBH}$, and $V_{\max }$ is the velocity at that point, then the angular momentum is $l=p V_{0}=r_{\min } V_{\max }$. The reduced mass of the BBH-star system is $\mu \sim m_{*}$, assuming the BHs to be much more massive than the stars. Then by conservation of energy,

$$
E=\frac{1}{2} m_{*} V_{\max }^{2}-\frac{G\left(m_{1}+m_{2}\right) m_{*}}{r_{\min }}=\frac{1}{2} m_{*} V_{0}^{2}
$$

Eliminating $V_{\max }$ and rearranging,

$$
p^{2}=r_{\min }^{2}+\frac{2 G\left(m_{1}+m_{2}\right)}{V_{0}^{2}} r_{\min }
$$

We set $r_{\min }=a$, the semi-major axis of the BBH, implying that an encounter occurs (i.e., according to our picture, the star interacts with the BBH and gets thrown out by the slingshot effect, while $a$ shrinks) if $p^{2} \leq a^{2}+\frac{2 G\left(m_{1}+m_{2}\right)}{V_{0}^{2}} a$. 
The number of stars per unit volume with velocity $v_{a}$ is $f\left(v_{a}\right) d^{3} v_{a} / m_{*}$. Hence, the number of encounters per unit time is

$$
\frac{1}{t_{e n}}=\int \frac{f\left(v_{a}\right)}{m_{*}} d^{3} v_{a} \pi p^{2} V_{0}
$$

where $V_{0}=\left|\vec{v}-\vec{v}_{a}\right|, \vec{v}$ being the velocity of the BBH. In order to take into account the wandering of the BBH's center of mass, this expression must be averaged over $\vec{v}$ (for a similar calculation, see Binney \& Tremaine [1987], §8.5). It was shown in Chatterjee, Hernquist \& Loeb (2002a, b) that the distribution of each component of $\vec{v}$ is Gaussian, so that the distribution of the magnitude, $v$, is $W(v)=\frac{2 v^{2}}{\sqrt{2 \pi} \sigma_{b h}^{3}} e^{-v^{2} / 2 \sigma_{b h}^{2}}$, with $\sigma_{b h}^{2}=\frac{2 G M m_{*}}{9 r_{0}\left(m_{1}+m_{2}\right)}$. Thus, averaging over $W$,

$$
\frac{1}{t_{e n}}=\frac{2 \pi}{\sqrt{2 \pi} m_{*} \sigma_{b h}^{3}} \int v^{2} e^{-v^{2} / 2 \sigma^{2}}\left(a^{2}+\frac{2 G\left(m_{1}+m_{2}\right)}{V_{0}^{2}} a\right) V_{0} f\left(v_{a}\right) d^{3} v_{a} d v
$$

or

$$
\frac{1}{t_{\text {en }}}=\frac{2 \pi}{\sqrt{2 \pi} m_{*} \sigma^{3}} \int v^{2} e^{-v^{2} / 2 \sigma^{2}}\left(a^{2}\left|\vec{v}-\overrightarrow{v_{a}}\right|+\frac{2 G\left(m_{1}+m_{2}\right)}{\left|\vec{v}-\overrightarrow{v_{a}}\right|} a\right) A\left(\psi-v_{a}^{2} / 2\right)^{7 / 2} d^{3} v_{a} d v
$$

where $A=\frac{96}{7 \sqrt{8} \pi^{3}} \frac{M r_{0}^{2}}{(G M)^{5}}$. Note that in the core, $\psi$ obtains an approximately constant value, $\psi \sim G M / r_{0}$.

The above integral can be simplified using the approximation $\left|\vec{v}-\overrightarrow{v_{a}}\right| \sim v_{a}$, since the BBH moves slowly relative to the stars when its mass is much larger than that of an individual star. The integration then gives,

$$
\frac{1}{t_{\text {en }}}=\frac{32 \pi^{2} A \psi^{11 / 2}}{99 m_{*}}\left(a+\frac{11}{2} \frac{G\left(m_{1}+m_{2}\right)}{\psi}\right) a .
$$

The BBH's binding energy is $E=\frac{G m_{1} m_{2}}{2 a}$. If each star on average extracts energy $\Delta E$, where $\Delta E=G \mu_{12} m_{*} / a$, is roughly the potential energy of the BBH-star system at the point of closest approach (giving $\Delta E / E=\frac{2 m_{*}}{m_{1}+m_{2}}$ ), then

$$
\frac{\Delta E}{\Delta t}=\frac{G m_{1} m_{2} m_{*}}{m_{1}+m_{2}} \frac{32 \pi^{2} A \psi^{11 / 2}}{99 m_{*}}\left(a+\frac{11}{2} \frac{G\left(m_{1}+m_{2}\right)}{\psi}\right)=-\frac{G m_{1} m_{2}}{2 a^{2}} \frac{d a}{d t}
$$

and simplifying,

$$
-\frac{1}{a^{2}\left(a+\frac{11 G\left(m_{1}+m_{2}\right)}{2 \psi}\right)} \frac{d a}{d t}=\frac{64 \pi^{2} A \psi^{11 / 2}}{99\left(m_{1}+m_{2}\right)} .
$$

Thus, there are two limiting regimes for the temporal evolution of $a$ : 
- If $a \gg 11 G\left(m_{1}+m_{2}\right) / 2 \psi$, then $\frac{-1}{a^{3}} \frac{d a}{d t}=\frac{C}{m_{1}+m_{2}}$, where $C$ is a constant, and we get $1 / a \propto t^{1 / 2}$.

- If $a \ll 11 G\left(m_{1}+m_{2}\right) / 2 \psi$, then $\frac{d}{d t}\left(\frac{1}{a}\right)=$ a constant, and we get $1 / a \propto t$.

For intermediate values, we get a power law, $1 / a \propto t^{d}$, with $0.5 \leq d \leq 1.0$.

Now, $11 G\left(m_{1}+m_{2}\right) / 2 \psi=\frac{11}{2} \frac{m_{1}+m_{2}}{M} r_{0}$. The BBH becomes hard at $a_{h} \sim \frac{3}{2} \frac{m_{2}}{M} r_{0}$, where $m_{2}$ is the lighter of the two BHs. Thus, after the BBH becomes hard, we always tend to the second case above, $1 / a \propto t$.

In our numerical experiments (to be described in the next section), we have found temporal power-law indices for $1 / a$ which are always bounded between $\sim 0.5$ (for heavy BBHs) and 1 (for light BBHs). The above simplified theory predicts a power-law index of 1 for hard binaries. However, our experiments showed that heavy BBHs (which contain more than a few percent of the mass of the Plummer galaxy) cause the central density of the galaxy to drop quickly, and so it is not appropriate to assume, as we did above, that the stellar distribution function remains unaffected by the BBH. In particular, the left-hand-side of equation (11) needs to be $\frac{-1}{a^{2}\left(a+c_{1}\right)} \frac{d a}{d t}$, where $c_{1}$ is now some undetermined term. If $c_{1}$ is not small compared to $a$, then it is possible to get $1 / a \propto t^{d}$, with $d<1$. This description is suggestive for why we obtained such limiting behaviors, and it is due to the form of the expression (5) for the impact parameter being a sum of two terms.

For low mass BBHs, the distribution function is not much affected by the hardening of the binary, and so we may proceed to derive the temporal slope of the $1 / a$ line,

$$
s=\frac{d}{d t}\left(\frac{1}{a}\right)=\frac{256 \sqrt{2}}{21 \pi}\left(\frac{G M}{r_{0}^{5}}\right)^{1 / 2} .
$$

Note that this result is independent of the masses of the two BHs.

We may compare the above value with the hardening rate presented in other work in a slightly different form: $\frac{d}{d t}\left(\frac{1}{a}\right)=\frac{G \rho_{0} H}{\sigma}$, where $H$ is a dimensionless hardening rate, $\rho_{0}$ is the central stellar density, and $\sigma$ is the one-dimensional stellar velocity dispersion in the core. Comparing with the above equation, we obtain $H=9.4$. This is very similar to the hardening rate observed in simulations by Milosavljević \& Merritt (2001). It also compares well with the value $H \sim 15$ (independent of $\mathrm{BH}$ mass ratio and orbital eccentricity), which is the hardening rate derived from three-body scattering experiments in the limit of a very hard binary (Quinlan 1996, Hills 1983).

In our simulations, we made the choice $r_{0}=3 \pi / 16$ and $G M=1$, for which one gets $s=\frac{d}{d t}\left(\frac{1}{a}\right)=20.6$. Note, however, the approximations that have been made in the derivation. 
We have assumed that the distribution function of the stars remains unchanged, which is clearly false: the central density of the stellar system falls, as shown in the next section.

For low mass BBHs (say $m<0.01$ ), however, the decline is not as much as for heavy BBHs, and we expect the inverse semi-major axis of the $\mathrm{BBH}$ to increase at a constant rate, with $s$ given roughly by the expression above.

We have assumed $\Delta E / E=2 m_{*} /\left(m_{1}+m_{2}\right)$, a form which is in reasonably good agreement with results of scattering experiments at several mass ratios (see Roos 1988, Hills 1983). It is, of course, an expression for the average energy extracted by a passing star, and need not accurately reflect the actual process of energy extraction for all impact parameters. Given that the precise form of this expression suitable for use in the massive BBH case is uncertain (for example, Hills \& Fullerton [1980] find that for equal-mass binaries, the right-hand side of the above expression may be multiplied by 0.7 ), it is perhaps optimistic to expect this derivation to give the correct slope $s$.

\section{Results from Numerical Experiments}

The numerical simulations were carried out using the SCFBDY program, which is described in detail in Quinlan \& Hernquist (1997) and Chatterjee, Hernquist \& Loeb (2002a). In our code, each BH interacts with the other BH and the stars through the $1 / r^{2}$ force, but stars interact with other stars through a mean field expansion of the gravitational potential that is updated self-consistently with time (note that the simulation thus does not treat star-star relaxation accurately [see Hernquist \& Barnes 1990]; this is justified since the two-body relaxation timescale in real galaxies is much longer than the Hubble time). The particles are moved with individual step-sizes using the fourth-order integrators of Aarseth (1994): NBODY1 for the stars and NBODY1 or NBODY2 for the BHs.

The mean field expansion of the gravitational potential is carried out in a set of basis functions that includes both spherical and non-spherical terms (Hernquist \& Ostriker 1992). However, since the galaxy remained nearly spherical during the integration, it made not much difference to the hardening of the BBH whether a few or zero non-spherical terms were included in the potential expansion.

We verified that numerical relaxation was not an important factor in our simulations by forcing the stars to interact only with a fixed background potential, and not with each other, and finding our results to be similar to those obtained using the full SCFBDY code. The insignificance of numerical relaxation in our simulations is further supported by experiments in which we forced the center of mass of the BBH to remain fixed, as described 
below.

Our results are presented in a system of units in which $G=M=1$, and $r_{0}=3 \pi / 16$, so that the energy of the initial galaxy without the BHs is $-1 / 4$ (Heggie \& Mathieu 1986). The stars (of equal mass $m_{*}=1 / N$, where $N$ is the number of stars) are initially distributed according to the Plummer distribution function, and the black holes are started out on nearly circular orbits at $\vec{r}$ and $-\vec{r}$, with $r=0.3$. (We tried a range of initial conditions for the BHs, including elliptical orbits; it made no difference to the hardening of the binary.) We have done a range of experiments, with binary mass ranging from $m_{1}=m_{2}=0.00125$ to $m_{1}=m_{2}=0.01$, and with particle numbers of up to $N=400,000$.

Figure 1 shows the results of such an integration with $m_{1}=m_{2}=0.00125$, and 400,000 stars. Shown are the separation between the BHs, $R$, the inverse semi-major axis of the $\mathrm{BBH}$ orbit, $1 / a$, the eccentricity of the orbit, $e$, and the central density, $\rho$, of the stellar system averaged over a sphere containing a mass in stars somewhat larger than the mass of the BBH. The BHs become bound at $t=77.3$. The BBH becomes hard once $a$ shrinks to the value $a_{h}=G m_{2} / 4 \sigma^{2}=1.104 \times 10^{-3}$, or when $1 / a_{h}=905$. As can be seen, the rate of change of $1 / a$ is nearly constant after the binary has become hard; namely, $1 / a \propto t^{d}$ with $d \simeq 1$.

Figure 2 shows the results of a similar integration with $m_{1}=m_{2}=0.02$, and 200,000 stars. The BHs become bound at $t=4.4$, and the BBH becomes hard when $a=a_{h}=0.018$, or when $1 / a=1 / a_{h}=56.6$. The rate of hardening is clearly not constant after this stage. Indeed, this is at the opposite extreme from the previous case, with the hardening being well fitted by $1 / a \propto t^{d}$ with $d \sim 0.5$.

For intermediate values of $m_{1}$ and $m_{2}$, the hardening occurs at the rate $1 / a \propto t^{d}$ with $0.5 \lesssim d \lesssim 1.0$. We have not found values of $d$ significantly outside this range. This is consistent with the explanation given in $\S 2$. Notice that for $m_{1}=m_{2}=0.00125$, the density at the position of the $\mathrm{BBH}$ center of mass drops gently over the course of its hardening. This is expected since the BBH expels stars from the center as it hardens. The magnitude of the drop is small, however, and thus to a first approximation, the stellar distribution function remains unaffected. On the other hand, the heavy BBH changes the distribution function to a greater extent, as is clear from the bottom right panel of Figure 2. Thus, in the former case, the theory developed in the previous section holds, and the binary hardens at a constant rate; in the latter case, we get a qualitatively different behavior, for the reasons outlined in $\S 2$.

The rate of hardening over time is close to constant in three cases for which we ran simulations: $m_{1}=m_{2}=0.00125, m_{1}=m_{2}=0.0025$, and $m_{1}=m_{2}=0.005$. Like Quinlan 
\& Hernquist (1997), we find that the slope of the $1 / a$ line, given by $s=\frac{d}{d t}(1 / a)$, varies with the number of stars. As the number of stars is increased, $s$ falls systematically, until there are roughly 200,000 - 400,000 stars, when it stabilizes to a particular value, $s_{0}$. This decrease in the slope as the number of stars increases has also been observed by Makino (1997) and Hemsendorf, Sigurdsson \& Spurzem (2002), though not by Milosavljević \& Merritt (2001). Quinlan \& Hernquist (1997) explained it as resulting from two competing factors governing the wandering of the BBH's center of mass: on the one hand, as the number of particles, $N$, rises, the binary wanders less from the center, and thus it reduces the density at the center more, and the hardening rate decreases; on the other hand, the restoring force of the stellar potential goes down as the central stellar density drops, causing an increase in the BBH's wandering. At a particular value of $N$, there is a balance between these two factors, and the hardening rate stops changing for higher values of $N$.

Quite apart from the variation of $s$ with $N$, we found that the limiting value of $s$ changes also with the mass of the BBH. We found that for $m_{1}=m_{2}=0.005, s$ stabilizes to a value of $s_{0} \sim 8$; for $m_{1}=m_{2}=0.0025, s_{0} \sim 11$; and for $m_{1}=m_{2}=0.00125, s_{0} \sim 15$. Recall that $s=20.6$ according to the simplified theory of $\S 2$. That value is expected to be obtained in the limit that the stellar distribution function remains unchanged by the influence of the binary. Our numerical experiments indeed reveal that this becomes a better approximation as the binary mass is reduced. However, the question remains whether this value is actually reached for still less massive BBHs.

The orbital eccentricity of the $\mathrm{BBH}$ in its hard state varies according to the mass of the binary. For massive binaries, such as for the case $m_{1}=m_{2}=0.02$ (Figure 2), the BBH orbits are circular to a good approximation. For smaller masses of the BBH, the eccentricity is larger: e.g., for $m_{1}=m_{2}=0.00125$ (Figure 1), the eccentricity in the hard state is roughly 0.3 , and trending very slightly upward. These values are consistent with Quinlan \& Hernquist (1997) and Milosavljević \& Merritt (2001), and with the results of scattering experiments in the restricted three-body approximation (Quinlan 1996), according to which the $\mathrm{BBH}$ eccentricity hardly grows if the initial eccentricity is moderate $\left(e_{0} \leq 0.3\right)$, and does not grow by much if it is larger, before the gravitational radiation stage sets in. However, Hemsendorf, Sigurdsson \& Spurzem (2002) and Aarseth (2002) have recently found that the eccentricity may grow to values close to 1 , resulting in rapid coalescence by the emission of gravitational radiation (see $\S 5$ ). The reason for this discrepancy is not clear, and may have to do with the initial conditions chosen for the simulations.

It is of interest to know the extent of wandering of the BBH. According to the formulae developed in Chatterjee, Hernquist \& Loeb (2002 a, b), the mean squared position and velocity of the center of mass of the $\mathrm{BBH}$ in the hard stage should, for the case of the 
Plummer potential, be given, respectively, by

$$
\begin{gathered}
r_{c m}^{2}=\frac{2 r_{0}^{2} m_{*}}{3\left(m_{1}+m_{2}\right)}, \\
v_{c m}^{2}=\frac{2 G M m_{*}}{3 r_{0}\left(m_{1}+m_{2}\right)} .
\end{gathered}
$$

This assumes that the stellar distribution function is unchanged, which is not the case for a massive $\mathrm{BBH}$; in fact, the wandering should be larger than indicated by these values, since the restoring force on the $\mathrm{BBH}$ drops as the central stellar density is diluted by the $\mathrm{BBH}$. However, the above expressions provide a reasonable description for the case of light BBHs. Indeed, we find from simulations that for such cases, the wandering is consistently larger than the values provided by the above formulae, but not by more than a factor of $\sim 2$ (see Table 1 ). The reasons for the enhanced wandering could still be the somewhat reduced stellar density at the center of the cluster, as well as the enhanced random motion of a binary compared with that of a point mass, as discussed by Merritt (2001). Thus, the binary is able to interact with a larger region of stellar phase space than calculated according to the simple model of $\S 2$.

\section{The Impact of Wandering on the Hardening of the BBH}

Quinlan \& Hernquist (1997) reported an experiment in which the center of mass of the binary was held fixed at the origin by the application of an artificial constraint force: they found that hardening halted soon after the binary became hard, following the evacuation of the loss cone. We have confirmed this result in our own experiments (see Figure 2 for an example): the $\mathrm{BBH}$ becomes hard when its semimajor axis reaches $a_{h}$, and continues hardening by three-body interactions with the stars until $a=a_{l c}\left(a_{l c}<a_{h}\right)$, when the loss cone is empty. These experiments show the importance of wandering in keeping the $\mathrm{BBH}$ hardening. (Incidentally, the fact that hardening stops in this case is another indication that numerical relaxation is not causing spurious refilling of the loss cone in our simulations.)

According to traditional theory (e.g., Begelman, Blandford and Rees [1980]), hardening of the binary should stop after the BBH has interacted with all the stars in its loss cone, as long as its wandering is unimportant. However, there is no sign in our experiments that a "hole" develops in the density distribution around the binary as it hardens - instead, the central density drops slowly as the binary hardens (see Figure 1). On the contrary, as

explained in the previous section, the wandering of the $\mathrm{BBH}$ increases as the central density falls, allowing it to interact with new regions of stellar phase space, and to keep hardening at a rate consistent with $1 / a \propto t^{d}$. The value of $d \approx 1$ is realized in the limit where the 
$\mathrm{BBH}$ makes up a small fraction of the host stellar system mass, as is typically the case for galactic bulges.

What if the mean wandering radius of the $\mathrm{BBH}$ were smaller than the semi-major axis at which the binary becomes hard $\left(a_{h}\right)$ ? Then the binary would wander "within itself", so to speak; in this case one may wonder whether it would not encounter fresh regions of phase space, and perhaps hardening would stop (the bottleneck, if there is one, would occur at the stage at which the binary becomes hard, because wandering only becomes more important as the semi-major axis of the binary shrinks). Is there a sign of this happening in the simulations?

Unfortunately, to do this experiment in the regime in which we are most interested (i.e., for BBHs of low mass, so that the hardening rate is constant over time), one would require the particle number to be prohibitively high. However, it is possible to perform this experiment for more massive BBHs. For example, Table 2 lists the experimental root-mean-square value of the binary's Brownian motion, $r_{c m}$, for a number of experiments, with the corresponding value of $a_{h}$; in each of the cases, $r_{c m}<a_{h}$. Hardening does not stop at or near this point, but continues indefinitely beyond this value at the rate $1 / a \propto t^{d}$, with $0.5 \lesssim d \lesssim 1.0$. Figure 2 shows the hardening of a BBH with $m_{1}=m_{2}=0.02$, with 200,000 particles. The rms value of the binary's Brownian motion is 0.013 ; the binary becomes hard when $a=a_{h}=3 m_{2} a / 2 M=0.018$, or when $1 / a_{h}=56$.

In each of these cases, however, we find that $r_{c m}>a_{l c}$; moreover, the value of $r_{c m}$ is very different from the value predicted by equation (13). This indicates that when the binary is allowed to wander, hardening no longer stalls at the semimajor axis $a_{l c}$; rather, the amplitude of its wandering increases as the central density of the star cluster declines (see Figure 3 for an explicit example of this), resulting in a lower restoring force holding the binary at the center. This, in turn, allows the binary to keep hardening by interacting with new stars that it would not have encountered if it did not wander. Thus, the "loss cone" is repopulated at a rate higher than the two-body relaxation rate by virtue of the increased wandering of the BBH's center of mass.

\section{Implications for the Coalescence of Massive Black Hole Binary Systems}

We have evidence, therefore, that the $\mathrm{BBH}$ can continue to harden beyond the point at which the loss cone would be evacuated were the binary's center of mass to be fixed at the origin, and that, moreover, it does so at roughly the rate calculated in $\S 2$. If we assume that hardening can continue until the point at which the emission of gravitational 
radiation becomes the dominant mode of energy loss for the binary, we can calculate when that should occur.

From equation (12), the hardening timescale of the BBH is

$$
t_{h}(a)=\left|\frac{a}{\dot{a}}\right|=\frac{21 \pi}{256 \sqrt{2}}\left(\frac{r_{0}^{5}}{G M}\right)^{1 / 2} \frac{1}{a} .
$$

The hardening timescale for gravitational radiation is (Peters 1964)

$$
t_{h}(a)=\left|\frac{a}{\dot{a}}\right|=\frac{5}{64} \frac{c^{5} a^{4}}{G^{3} \mu_{12}\left(m_{1}+m_{2}\right)^{2}} \frac{\left(1-e^{2}\right)^{7 / 2}}{1+73 e^{2} / 24+37 e^{4} / 96},
$$

where $c$ is the velocity of light, and $e$ the eccentricity of the BBH's orbit. The dependence of the gravitational radiation timescale on eccentricity is weak unless $e$ is close to 1 . As noted in $\S 3, e$ is very small for heavy BBHs, but rises to larger values $(\sim 0.2)$ for lighter BBHs. In the absence of a precise characterization of the variation of $e$ with $\mathrm{BBH}$ mass, we take $e=0$ in the above expression; thus, our result for the time of onset of gravitational wave emission and coalescence should be regarded as an upper limit, as a higher eccentricity would allow this stage to be reached more quickly.

Equating the two expressions, we have that the semi-major axis at which gravitational radiation losses start to dominate, $a_{g r}$, is given by

$$
a_{g r}^{5}=\frac{21 \pi}{20 \sqrt{2}} \frac{G^{5 / 2} m_{1}^{3} q(1+q) r_{0}^{5 / 2}}{c^{5} M^{1 / 2}},
$$

where $q$ is the mass ratio $q=m_{2} / m_{1} \leq 1$.

From equation (12), assuming that $a_{g r} \ll a_{h}$, we can derive the time between the binary's becoming hard at semi-major axis $a_{h}$, and the time at which $a=a_{g r}$,

$$
t_{g r}=\frac{21 \pi}{256 \sqrt{2}} \frac{r_{0}^{5 / 2}}{G^{1 / 2} M^{1 / 2}} \frac{1}{a_{g r}}
$$

or

$$
t_{g r}=\frac{21 \pi}{256 \sqrt{2}}\left(\frac{20 \sqrt{2}}{21 \pi}\right)^{1 / 5} \frac{c r_{0}^{2}}{G M^{2 / 5} m_{1}^{3 / 5}[q(1+q)]^{1 / 5}} .
$$

The dependences on $M, m_{1}$ and $q$ are rather weak.

We can also calculate the total number of stars, $N_{\text {int }}$, the BBH must interact with from the time it becomes hard to the time $t_{g r}$. We have $\Delta E=G \mu_{12} m_{*} / a=\frac{G m_{*}}{a} \frac{m_{1} q}{1+q}$. Therefore $d E=\frac{G m_{*}}{a} \frac{m_{1} q}{1+q} d N$, or $\frac{d E}{d N}=\frac{G m_{*}}{a} \frac{m_{1} q}{1+q}$. But $E=G m_{1} m_{2} / 2 a$, and so $\frac{d E}{d N}=\frac{-G m_{1}^{2} q}{2 a^{2}} \frac{d a}{d N}$. Equating 
the above, we get $\frac{d N}{d a}=-\frac{m_{1}}{m_{*}} \frac{1+q}{2 a}$. Integrating, the total number of stars the BBH interacts with is

$$
N_{\text {int }}=\frac{m_{1}(1+q)}{2 m_{*}} \ln \left(\frac{a_{h}}{a_{g r}}\right)
$$

where $a_{h}=3 m_{2} r_{0} / 2 M=3 m_{1} q r_{0} / 2 M$. The total mass with which the BBH interacts is then simply

$$
M_{i n t}=\frac{\left(m_{1}+m_{2}\right)}{2} \ln \left(\frac{a_{h}}{a_{g r}}\right) .
$$

As will be seen from Figure 4c, the total mass in stars the BBH must interact with is roughly of the order of itself. Therefore, according to this theory, the BBH should not change the stellar distribution function much, as long as the mass of the BBH is not significant in comparison with the total mass in stars. This result is consistent with our simulations for small mass BBHs.

Some of the above relations are depicted for physically reasonable stellar systems and BBHs in Figure 4. Instead of presenting results in terms of $M$ and $r_{0}$, we use more physically and observationally relevant variables, such as the one-dimensional stellar velocity dispersion, $\sigma$, and the central density of the stellar system, $\rho_{0}$. The relations amongst these quantities for the Plummer model are as follows:

$$
\begin{gathered}
\rho_{0}=\frac{3 M}{4 \pi r_{0}^{3}}, \\
\sigma^{2}=\frac{G M}{6 r_{0}} .
\end{gathered}
$$

We then obtain

$$
a_{g r}^{5}=\frac{63 \sqrt{3}}{80} \frac{G^{2} m_{1}^{3} q(1+q) \sigma}{c^{5} \rho_{0}}
$$

and

$$
t_{g r}=\frac{63 \sqrt{3}}{1024}\left(\frac{80}{63 \sqrt{3}}\right)^{1 / 5} \frac{c \sigma^{4 / 5}}{G^{7 / 5} m_{1}^{3 / 5}[q(1+q)]^{1 / 5} \rho_{0}^{4 / 5}} .
$$

Figures $4 \mathrm{a}$ and $4 \mathrm{~b}$ show that for physically reasonable masses of the $\mathrm{BBH}$ and characteristics of the host galaxy, the semi-major axis can shrink to the value $a_{g r}$ within a Hubble time, after which coalescence occurs quickly by the emission of gravitational waves. The actual values of $a_{g r}$ are very small (Figure $4 \mathrm{~d}$ ).

The above calculations assume that the binary hardens according to $1 / a \propto t$, which is the behavior we see in the simulations when the mass of the $\mathrm{BBH}$ is less than about $1 \%$ of the total mass in stars. Is this a reliable guide to what happens in actual galaxies? 
The ratio of the mass of the $\mathrm{BH}$ to the mass of the galaxy is observed to be quite low (on the order of $10^{-3}$ : see Merritt \& Ferrarese 2001, Wandel 1999, Magorrian et al. 1998), and therefore the linear hardening behavior is most likely to apply in the cases of practical interest.

However, it is interesting to ask what would happen if the BBH hardened more slowly than at a constant rate. As an example, consider the case shown in Figure 2, with $m_{1}=m_{2}=0.02$, and $N=200,000$ stars. The hardening behavior is well fit by the expression $1 / a=21.7 \times t^{0.5}$. For purposes of illustration, let us use $a_{g r_{1}}$ to denote the semimajor axis at which gravitational wave radiation becomes dominant if we assume that the $\mathrm{BBH}$ hardens at a constant rate, $1 / a \propto 20.6 \times t$, and $a_{g r_{2}}$ the semimajor axis at which gravitational wave radiation becomes dominant if we assume that the $\mathrm{BBH}$ hardens at the above non-linear rate, $1 / a \propto 21.7 \times t^{0.5}$, as is actually observed in the simulations. If we scale our galaxy such that $r_{0}=3 \pi / 16$ corresponds to $100 \mathrm{pc}$, and $M=1$ corresponds to a total mass in stars in the bulge of $10^{9} M_{\odot}$, then we obtain $a_{g r_{1}} / a_{g r_{2}} \simeq 0.3$. Thus, the gravitational radiation stage sets in at a larger semimajor axis if hardening occurs more slowly than linearly. This arises from the different behaviors of the hardening timescales: when $1 / a \propto t, t_{h}=|a / \dot{a}| \propto 1 / a$, whereas when $1 / a \propto t^{0.5}, t_{h} \propto 1 / a^{2}$. However, the times taken to reach $a_{g r_{1}}$ and $a_{g r_{2}}$ are approximately 1 Gyr and 70 Gyr respectively, since in the latter case the BBH hardens only as $t^{0.5}$ rather than $\propto t$, as in the former case. Therefore, despite the fact that the gravitational radiation stage is reached at a larger semimajor axis if hardening occurs slower than linearly, the time taken to reach this stage is longer in the former case. Thus, our results in this section hold for light BHs which, as mentioned before, is the case with most galaxies.

\section{Summary}

In summary, our primary results are:

- We have developed a consistent description for the behavior of the semi-major axis of a hard $\mathrm{BBH}$. As long as the BBH mass is small compared to the total mass in stars: $1 / a \propto t^{d}$, with $d \approx 1$.

- Our simplified theory gives the value of the proportionality constant of the above linear relation, $s=20.6$, independent of the mass of the BBH or the number of stars. We find, however, from numerical experiments that $s$ depends on the number of stars - as the number of stars increases, $s$ drops, but eventually converges to a particular value for $N \gtrsim 200,000$ stars. A possible explanation is that this reflects 
an equilibrium between two competing processes: as the number of stars increases, the wandering of the binary becomes smaller, causing a larger drop in central density; this, however, increases the wandering of the $\mathrm{BBH}$ because of the reduction of the central restoring force on it.

- We also find that $s$ depends on the mass of the BBH: in our simulations for equal-mass $\mathrm{BHs}$, the convergent value of $s$ increases from $\sim 8$ to $\sim 15$ as the mass of each $\mathrm{BH}$ drops from 0.005 to 0.00125 . We interpret this as meaning that the theoretical value 20.6 is reached when no change in the stellar distribution function is caused by the $\mathrm{BBH}$ - this will be true only when the mass of the BBH is very small compared to the total mass in stars. If we could run simulations with even lower mass BBHs, we may approach 20.6. However, it should be remembered that we have assumed a somewhat uncertain form for the energy removed from the binary by each stellar interaction. We also note that for calculating the time at which gravitational radiation starts to dominate the energy loss, we do not need to know the precise value of $s$, since the fifth root of this number needs to be taken, as shown in $§ 5$. There are two difficulties with running simulations with very light BBHs: first, the black holes need to be much more massive than the stars; second, the dynamical friction timescale for light black holes, and consequently the time for them to become hard, is very long.

- For light BBHs, strong disruption of the stellar density does not occur: the central density of the cluster does decline with time but gently. This is where we expect our theory to be applicable. There is no sign that a "hole" is forming at the center, and thus no sign that hardening would stop after the loss cone is evacuated. Wandering allows the BBH to interact with regions of stellar phase space it would not otherwise encounter. According to our experiments, if we keep the BBH center of mass artificially clamped to the center, hardening quickly stops after the BBH has interacted with the stars that could pass within roughly a distance $a$ from it.

- For heavy BBHs, there is stronger disruption of the stellar density and distribution function, and strong deviations from the linear behavior of the inverse semi-major axis: $1 / a \propto t^{d}$, with $0.5 \lesssim d \lesssim 1$. This is the regime in which our theory is not applicable, though it gives a qualitative explanation for why $d$ should be bracketed by 0.5 and 1 .

- We have shown that the hardening of the BBH does not stop even when its typical wandering radius is smaller than the value of the semi-major axis at which it becomes hard, at least in the case when the $\mathrm{BBH}$ is fairly massive; however, the wandering radius is always larger than the semi-major axis at which hardening would halt because of loss cone depletion if the binary did not wander. The reason involves the 
increased wandering of the binary's center of mass as the central density of the stellar system falls, resulting in a reduction of the restoring force keeping the binary at the center. To verify this in the case of light binaries would require simulations with many millions of particles.

- We have calculated an upper limit on the time required for the BBHs to reach the stage at which energy losses are dominated by gravitational radiation emission. For reasonable characteristics of the stellar system, the gravitational radiation stage is reached within a Hubble time. In order to reach this stage, the $\mathrm{BBH}$ must interact with a total mass in stars that is of order its own mass.

Our model uses the spherically symmetric Plummer density profile, which has a flat density structure at the center. However, observations have shown that most elliptical galaxies are characterized by weak or strong cusps (e.g., Faber et al. 1997, Byun et al. 1996, Lauer et al. 1995). Is it then reasonable to use the Plummer model in calculations of $\mathrm{BBH}$ coalescence?

The Plummer model is characterized by a simple distribution function, which makes it possible to obtain analytic results for the phenomena discussed in this paper. Here, we are mainly interested in understanding the effect of the binary's Brownian motion on its hardening, rather than in calculating precisely the time taken for a binary in a real galaxy to coalesce. Despite the fact that our model for galactic bulges is unrealistic, we believe that our results capture generic dynamical processes that take place in more complicated situations for a number of reasons. First, recent simulations have shown that the merger of two galaxies with steep density cusps produce a galaxy with a shallow density cusp (Milosavljević \& Merritt 2001), owing to the transfer of energy from the binary to the stars; moreover, the destruction of the steep cusp takes place very rapidly (on a timescale comparable to the local dynamical time), before the binary becomes significantly hard (similar results have been obtained by Nakano and Makino 1999 a, b). Our results apply to the stage when the binary has become hard. Secondly, the weak cusp of the merged galaxy is carried by the BBH as it wanders around; whereas the stellar density and velocity dispersion in our calculations refer to their values in the core of the galaxy but outside the Keplerian rise around the black holes, since most of the encounters that remove energy from the $\mathrm{BBH}$ are with stars from outside the cusp. Thirdly, the presence of the density cusp would only increase the supply of stars to the binary, resulting in more efficient hardening; if a binary coalesces within a Hubble time in our model, it would likely coalesce within a shorter time in the presence of a cusp. 
We thank G. Quinlan for providing the simulation code. For useful comments, we thank G. Rybicki and the participants of the Massive Black Hole Coalescence Focus Session at Pennsylvania State University, November 2002. AL acknowledges support from the Institute for Advanced Study at Princeton, the John Simon Guggenheim Memorial Fellowship, and NSF grants AST-0071019, AST-0204514.

\section{REFERENCES}

Aarseth, S.J. 2002, astro-ph/0210116

Aarseth, S.J. 1994, Direct Methods for N-Body Simulations, in Galactic Dynamics and N-Body Simulations, eds. G. Contopoulos, N.K. Spyrou \& L. Vlahos (Springer-Verlag)

Armitage, P.J. \& Natarajan, P. 2002, ApJ 567, L9

Begelman, M.C., Blandford, R.D. \& Rees, M.J. 1980, Nature 287, 307

Binney, J., \& Tremaine, S. 1987, Galactic Dynamics (Princeton: Princeton Univ. Press)

Byun, Y. et al. 1996, AJ 111, 1889

Chatterjee, P., Hernquist, L. \& Loeb, A. 2002a, ApJ 572, 371

Chatterjee, P., Hernquist, L. \& Loeb, A. 2002b, Phys. Rev. Lett. 88, 121103

Faber, S.M. et al. 1997, AJ 114, 1771

Frank, J. \& Rees, M.J. 1976, MNRAS 176, 633

Gould, A., \& Rix, H.-W. 2000, ApJ, 532, L29

Heggie, D.C. \& Mathieu, R.M. 1986, in P. Hut \& S.L.W. McMillan (eds.), The Use of Supercomputers in Stellar Dynamics, pp. 233-235, New York

Hemsendorf, M., Sigurdsson, S. \& Spurzem, R. 2002, astro-ph/0103410

Hernquist, L. \& Barnes, J. 1990, ApJ 349, 562

Hernquist, L. \& Ostriker, J.P. 1992, ApJ 386, 375

Hills, J.G. 1983, AJ 88, 1269

Hills, J.G. \& Fullerton, L.W. 1980, AJ 85, 1281

Lauer, T.R. et al. 1995, AJ 110, 2622

Magorrian, J., et al. 1998, AJ 115, 2285

Makino, J. 1997, ApJ 478, 58 
Merritt, D. 2001, ApJ 556, 245

Merritt, D. \& Ferrarese, L. 2001, MNRAS 320, L30

Nakano, T. \& Makino, J. 1999a, ApJ 525, L77

Nakano, T. \& Makino, J. 1999b, ApJ 510, 155

Milosavljević, M. \& Merritt, D. 2001, ApJ 563, 34

Milosavljević, M. \& Merritt, D. 2002, astro-ph/0212459

Quinlan, G.D. 1996, NewA 1, 35

Quinlan, G.D. \& Hernquist, L. 1997, NewA 2, 533

Peters, P.C. 1964, Phys. Rev. B 136, 1224

Roos, N. 1988, ApJ 334, 95

Wandel, A. 1999, ApJ 519, L39

Yu, Q. 2002, MNRAS 331, 935 


\begin{tabular}{|c|c|c|c|c|}
\hline$m_{1}=m_{2}$ & $N$ & $r_{c m}$ (theory) & $r_{c m}$ (expt.) & $a_{h}$ \\
\hline 0.00125 & 400000 & 0.0152 & 0.0251 & 0.011 \\
\hline 0.00125 & 200000 & 0.0215 & 0.0386 & 0.011 \\
\hline 0.0025 & 200000 & 0.0152 & 0.0298 & 0.022 \\
\hline 0.0025 & 100000 & 0.0215 & 0.0321 & 0.022 \\
\hline 0.005 & 200000 & 0.0108 & 0.0185 & 0.044 \\
\hline 0.005 & 100000 & 0.0152 & 0.0273 & 0.044 \\
\hline
\end{tabular}

Table 1: Values of the wandering radius, $r_{c m}$, of the binary's center of mass according to our simplified theory (equation [13]) and numerical experiments, and the corresponding values of $a_{h}$.

\begin{tabular}{|c|c|c|c|c|c|}
\hline$m_{1}=m_{2}$ & $N$ & $r_{c m}$ (theory) & $r_{c m}$ (expt.) & $a_{h}$ & $a_{l c}$ \\
\hline 0.02 & 200000 & 0.0054 & 0.0129 & 0.0177 & 0.008 \\
\hline 0.04 & 100000 & 0.0054 & 0.0202 & 0.0353 & 0.011 \\
\hline 0.05 & 50000 & 0.0068 & 0.0142 & 0.0442 & 0.013 \\
\hline 0.05 & 100000 & 0.0048 & 0.0321 & 0.0442 & 0.015 \\
\hline 0.10 & 50000 & 0.0048 & 0.0376 & 0.0884 & 0.021 \\
\hline 0.10 & 100000 & 0.0034 & 0.0444 & 0.0884 & 0.021 \\
\hline
\end{tabular}

Table 2: Values of the wandering radius, $r_{c m}$, of the binary according to our simplified theory (equation [13]) and numerical experiments, and the corresponding values of $a_{h}$ and $a_{l c}$. 

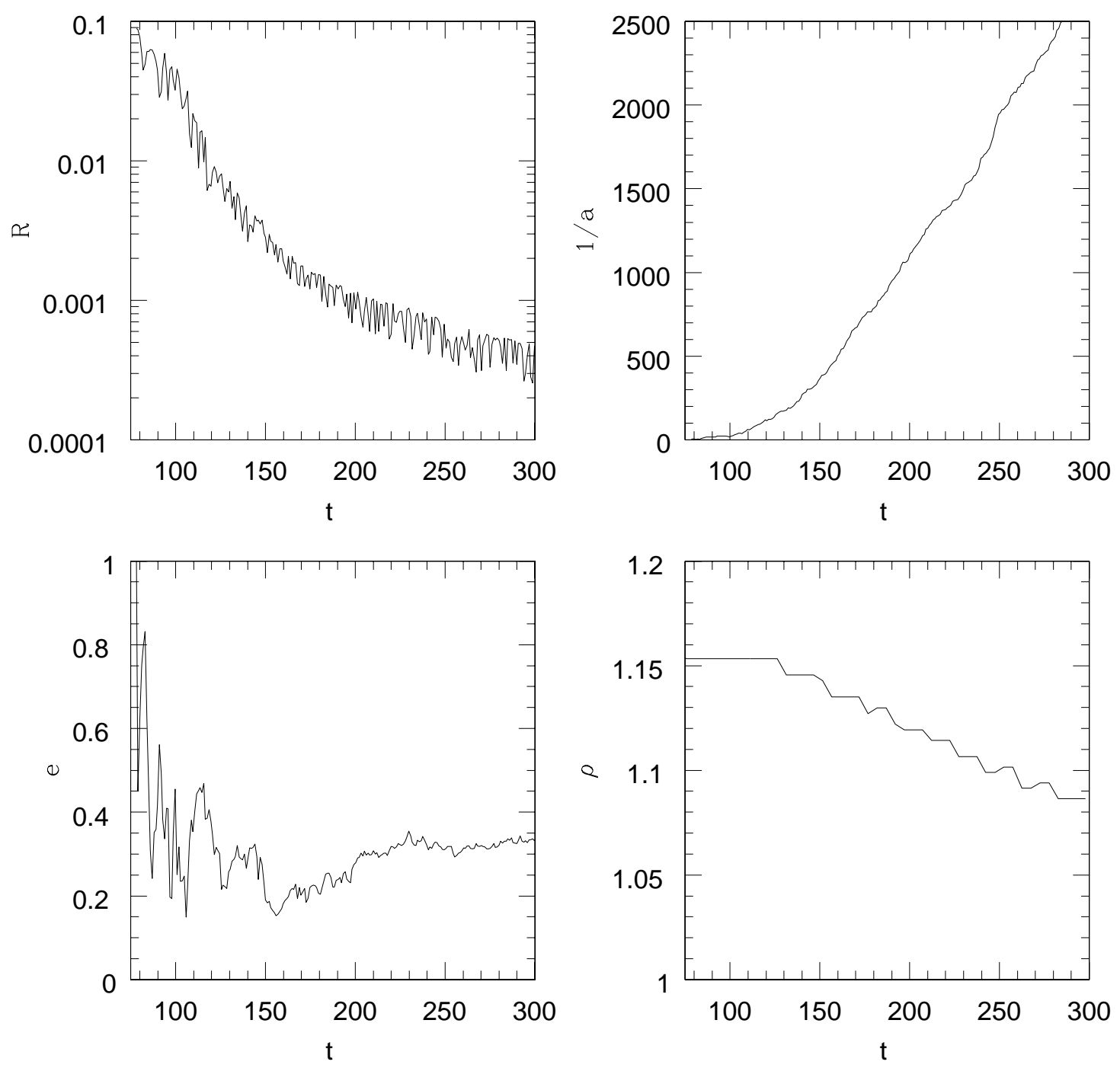

Fig. 1. - Results from a simulation of a binary black hole system with $m_{1}=m_{2}=0.00125$ and 400,000 stars. Shown are the black hole separation, $R$, the reciprocal of the semi-major axis of the binary's orbit, $a$, its eccentricity, $e$, and the density at the center of the potential, $\rho$. The binary becomes hard at $1 / a \simeq 1 / a_{h}=905$; it subsequently hardens at approximately a constant rate, $s=\frac{d}{d t}\left(\frac{1}{a}\right) \simeq 15.5$. 

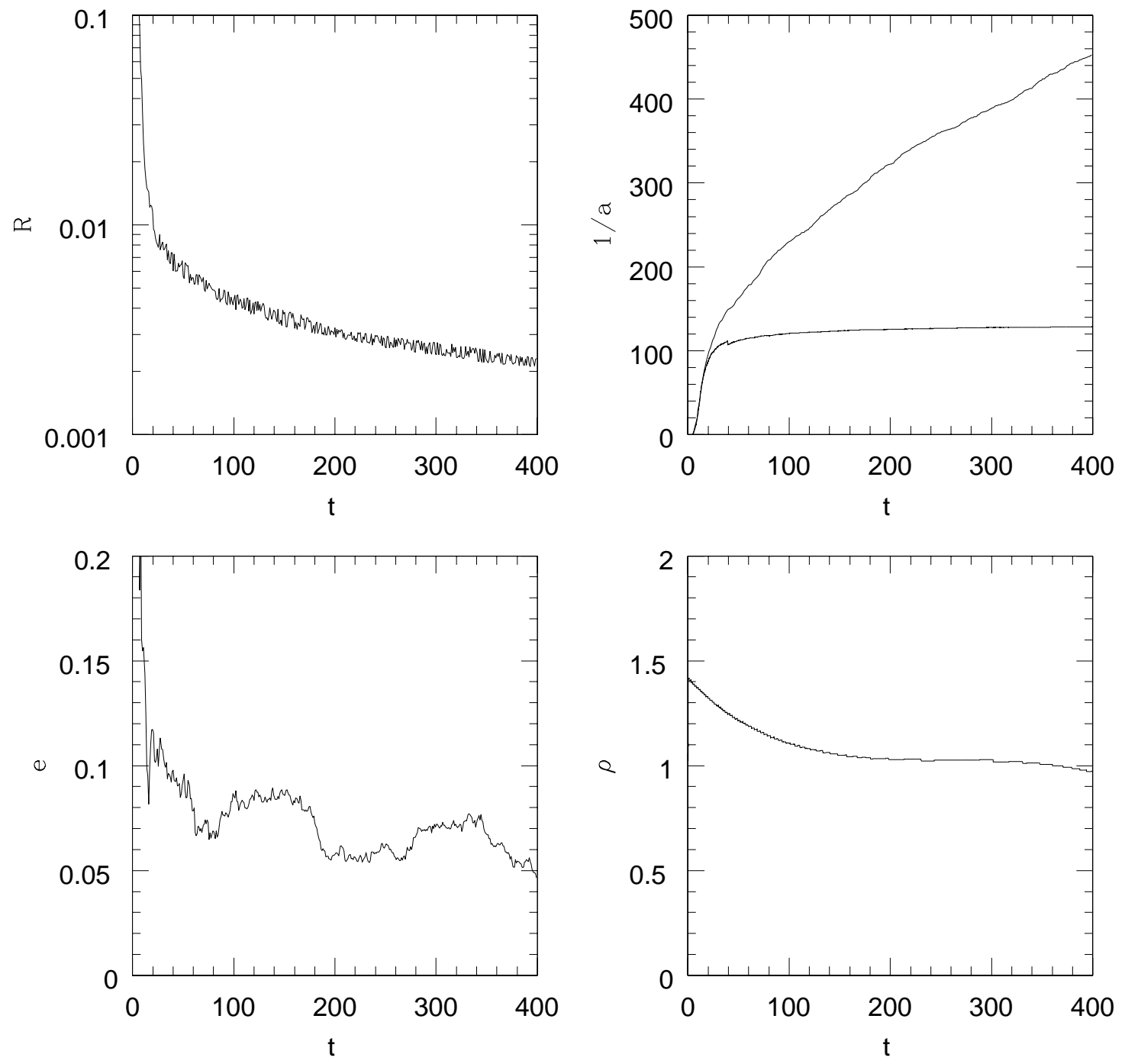

Fig. 2. - The same as Figure 1, except that $m_{1}=m_{2}=0.02$ and $N=200,000$. The binary becomes hard at $1 / a \simeq 1 / a_{h}=57$; it subsequently hardens at a rate given approximately by $1 / a \propto t^{d}$, with $d \sim 0.5$. If the center of mass of the binary is held fixed at the origin, hardening stops as $1 / a \simeq 1 / a_{l c}=130$, as indicated by the lower curve of the upper right panel. 

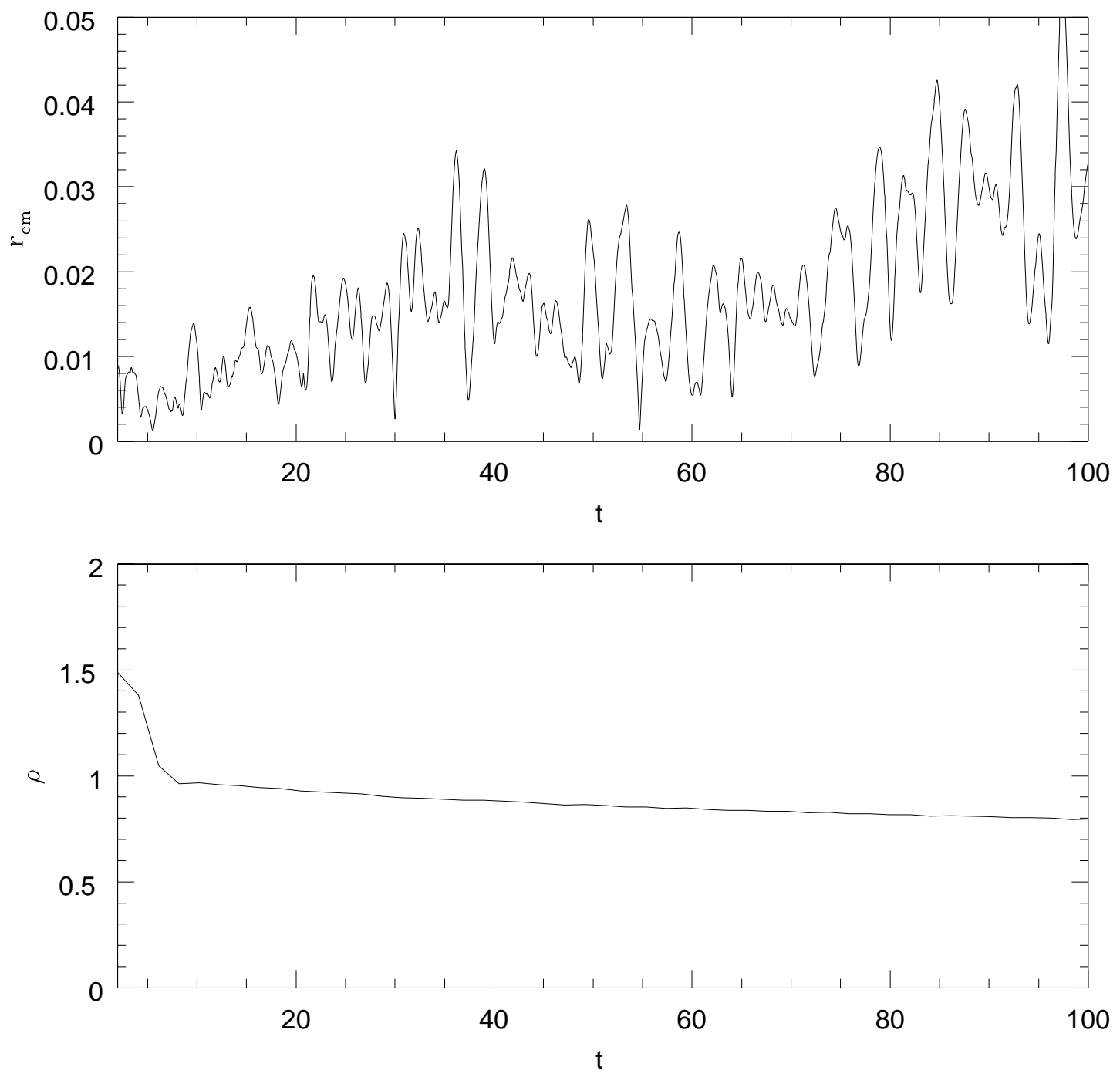

Fig. 3. - The radial distance of the binary's center of mass from the origin, and the density at the center of the potential, for a simulation with $m_{1}=m_{2}=0.04$ and $N=100,000$. The wandering of the BBH's center of mass increases as the central density drops. 

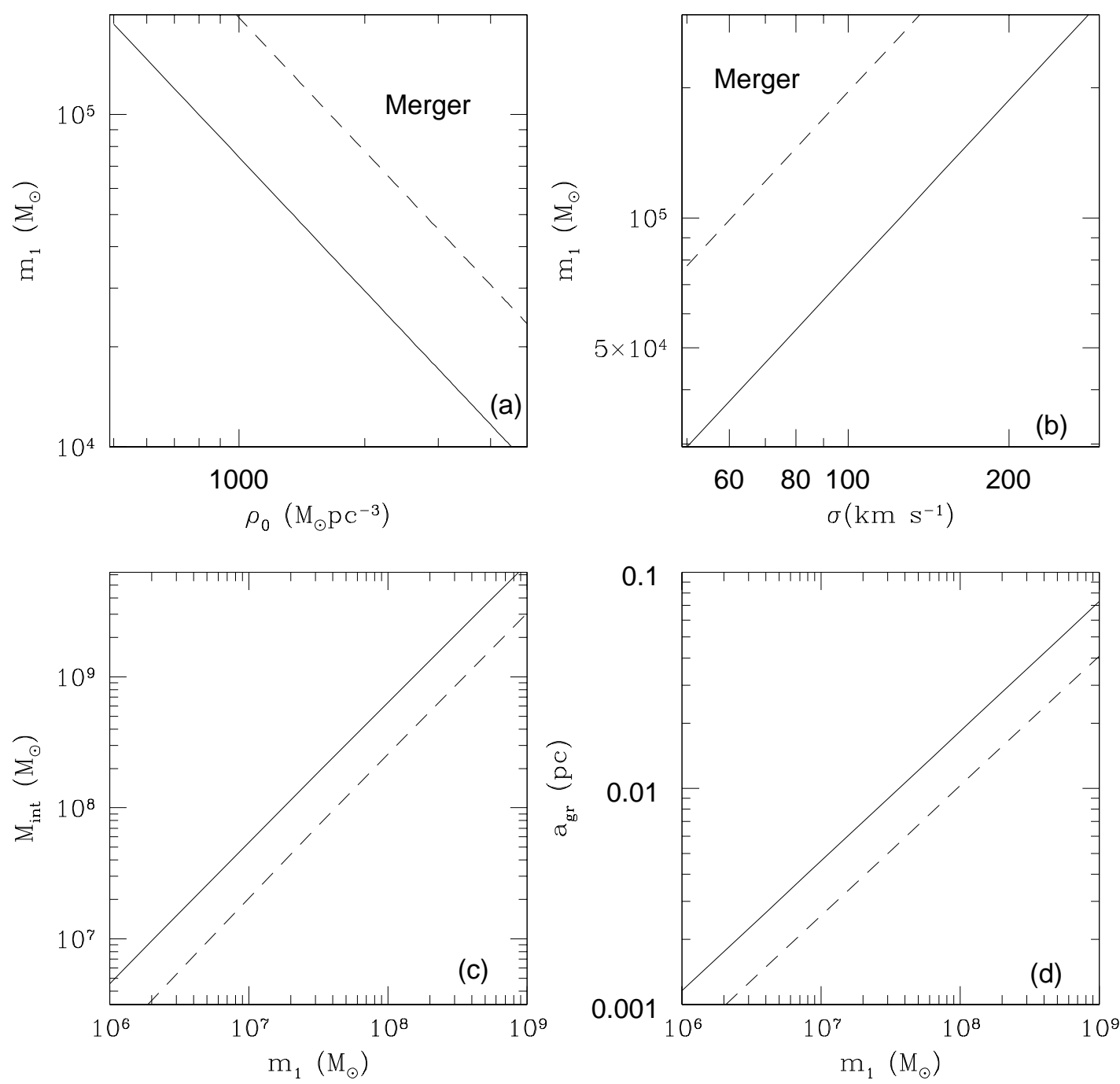

Fig. 4.- (a) The line indicates those values of $m_{1}$ and galaxy central density, $\rho_{0}$, for which the $\mathrm{BBH}$ reaches the gravitational wave stage $\left(a=a_{g r}\right)$ in $10 \mathrm{Gyr}$; points above this line correspond to combinations for which this stage is reached in less time. The stellar velocity dispersion $\sigma$ is set to $100 \mathrm{~km} / \mathrm{s}$. (b) The line indicates those values of $m_{1}$ and $\sigma$ for which the BBH reaches $a=a_{g r}$ in $10 \mathrm{Gyr}$; points above this line correspond to combinations for which this stage is reached in less time. $\rho_{0}=1000 M_{\odot} \mathrm{pc}^{-3}$. (c) The total mass in stars the binary must interact with until $a=a_{g r} ; \sigma=100 \mathrm{~km} / \mathrm{s}$ and $\rho_{0}=1000 M_{\odot} \mathrm{pc}^{-3}$. (d) $a_{g r}$ as a function of $m_{1} ; \sigma=100 \mathrm{~km} / \mathrm{s}$ and $\rho_{0}=1000 M_{\odot} \mathrm{pc}^{-3}$. The solid lines are for $m_{1}=m_{2}$, the dashed lines for $m_{1}=10 m_{2}$. 